\title{
Development of an Algorithm for the Diagnosis of Otitis Media
}

\author{
Nader Shaikh, MD, MPH; Alejandro Hoberman, MD; Howard E. Rockette, PhD; \\ Marcia Kurs-Lasky, MS
}

From the Division of General Academic Pediatrics, Children's Hospital of Pittsburgh of UPMC, University of Pittsburgh School of Medicine (Drs. Shaikh and Hoberman); and Department of Biostatistics, University of Pittsburgh Graduate School of Public Health (Dr. Rockette and Ms Kurs-Lasky), Pittsburgh, PA

The authors have no conflicts of interest to disclose.

Address correspondence to: Nader Shaikh, MD, MPH, Children's Hospital of Pittsburgh of UPMC, One Children's Hospital Drive,

4401 Penn Ave, Pittsburgh, PA 15224 (e-mail: nader.shaikh@ @ chp.edu).

Received for publication August 22, 2011; accepted January 19, 2012.

\section{Abstract}

BACKGROUND: The relative importance of signs and symptoms in the diagnosis of otitis media has not been adequately evaluated. This has led to a large degree of variation in the criteria used to diagnose otitis media, which has resulted in inconsistencies in clinical care and discrepant research findings. METHODS: A group of experienced otoscopists examined children presenting for primary care. We investigated the signs and symptoms that these otoscopists used to distinguish acute otitis media (AOM), otitis media with effusion (OME), and no effusion. We used recursive partitioning to develop a diagnostic algorithm. To assess the algorithm, we validated it in an independent dataset.

RESULTS: Bulging of the tympanic membrane (TM) was the main finding that otoscopists used to discriminate AOM from OME; information regarding the presence or absence of other signs and symptoms added little to the diagnostic process. Overall, $92 \%$ of children with AOM had a bulging TM compared with $0 \%$ of children with OME. Opacification and/or an airfluid level was the main finding that the otoscopists used to discriminate OME from no effusion; $97 \%$ of children diagnosed with OME had an opaque TM compared with $5 \%$ of children diagnosed with no effusion. An algorithm that used bulging and opacification of the TM correctly classified $99 \%$ of ears in an independent dataset.

Conclusions: Bulging of the TM was the finding that best discriminated AOM from OME. The algorithm developed here may prove to be useful in clinical care, research, and education concerning otitis media.

Keywords: acute otitis media; diagnostic algorithm; otitis media; otitis media with effusion; otoscopy; physical examination; signs and symptoms

Academic Pediatrics 2012;12:214-218

\section{What'S NEW}

We developed and validated an algorithm that describes the signs and symptoms that experienced otoscopists use when diagnosing otitis media. This algorithm promises to be useful in clinical care, research, and education concerning otitis media.

SYMPTOMS ARE CLEARLY important in the diagnosis and management of children with acute otitis media (AOM). The presence of symptoms prompts parents to seek medical care, and the subsequent course of symptoms helps guide clinical care for these children. Clinicians prescribe antimicrobials for children with $\mathrm{AOM}$ in part under the assumption that this results in a more rapid resolution of symptoms. In addition, symptoms (eg, otalgia) are often used to determine eligibility for clinical trials.

In several studies authors have identified symptoms that correlate with otoscopic diagnosis, but no studies to date have compared the relative importance of otoscopic signs versus symptoms in the diagnostic process. This absence of empiric data regarding the relative importance of signs and symptoms is partly responsible for that lack of consensus that exists regarding the diagnostic criteria for otitis media. For example, the 2004 American Academy of Pediatrics Guidelines for the diagnosis of AOM provided clinicians with a list of signs and symptoms associated with the diagnosis of AOM. ${ }^{1}$ However, without information regarding the relative importance of these symptoms with respect to each other, and with respect to signs present on otoscopic examination, such a list is difficult to implement in practice. This was best illustrated by a study by Hayden, who, in a survey of 165 pediatricians, found that 147 different combinations of signs and symptoms were endorsed as criteria for diagnosis. ${ }^{2}$ The consequences of inaccurate diagnosis are not trivial; inappropriate use of antimicrobials for AOM contributes significantly to increased resistance among respiratorytract pathogens. Furthermore, the use of nonstringent diagnostic criteria in clinical trials has permitted the inclusion of children who did not actually have AOM but instead had otitis media with effusion (OME) in conjunction with nonspecific symptoms. This has resulted in 
discrepant and misleading conclusions regarding the treatment of $\mathrm{AOM}^{3}$

By empirically examining the findings used by a group of experienced otoscopists to classify otitis media, we aimed 1) to determine the relative importance of signs and symptoms in the diagnosis of AOM, and 2) to use this information to develop a diagnostic algorithm that could categorize children into the 3 diagnostic categories, that is, AOM, OME, or no effusion. We assessed the performance of the algorithm by applying it to an independent dataset. Please see a related video at http://www. academicpedsjnl.net/content/acap-videos.

\section{METHODS}

We developed a decision tree by using data from a previously conducted cohort study (of pneumococcal colonization) in which a convenience sample of 264 children (mean age, 12.5 months) who presented for primary care or sick visits were followed for one respiratory season via the use of serial pneumatic otoscopic examinations. ${ }^{4}$ This dataset will be referred to as the training dataset. The study otoscopists had previously completed a training program in which their diagnoses had been validated against findings at myringotomy. ${ }^{5}$ At each visit, and after the removal of cerumen, an otoscopist assessed the following tympanic membrane (TM) characteristics: color (amber, blue, gray, pink, white, yellow), degree of opacification (translucent, semiopaque, opaque), position (neutral, retracted, bulging), decreased mobility (yes, no), presence of airfluid level(s) (yes, no), and presence of areas of marked redness (yes, no). At each visit, and before otoscopic examination, symptoms were recorded by the parent with a previously developed patient-reported outcome measure (Acute Otitis Media Severity of Symptoms Scale [AOMSOS]). ${ }^{4,6}$ The version of the AOM-SOS scale used in this study asked parents about the presence of 7 symptoms during the preceding 24 hours (ear pain, ear tugging, irritability, decreased play, decreased appetite, difficulty sleeping, and fever).

In children with AOM in one ear and OME or no effusion in the other, we selected the ear with AOM. In children with OME in one ear and no effusion in the other, we selected the ear with OME. In children with the same diagnosis in both sides, we arbitrarily chose the left ear. For each child, only the first visit with a complete set of signs and symptoms was presented. We repeated the analysis by using the right ear and using all possible visits. The results were essentially the same and thus will not be presented.

We first examined the univariate association between TM findings and the assigned diagnosis by using logistic regression. We then used recursive partitioning (CART 6.6, Salford Systems, San Diego, CA) to develop a decision tree that used signs and symptoms to classify cases into 1 of 3 diagnostic categories: AOM, OME, and no effusion. Recursive partitioning uses the predictor variables (eg, signs and symptoms) to repeatedly stratify the study group into mutually exclusive subgroups in a manner that categorizes the subjects by the outcome variable. Recursive partitioning was also used to determine the relative importance of each variable in the diagnostic process. The "importance score" from the recursive partitioning analysis represents the relative improvement in the final classification attributable to each variable (with the best variable assigned a relative value of 100). For the recursive partitioning analysis, we dichotomized predictor variables as follows: each symptom (present vs absent), position (bulging vs not bulging), air-fluid level or opacification (present vs absent), mobility (normal vs absent or decreased), color (gray or pink vs yellow or white or amber or blue), and marked redness (present vs absent). We used the default settings in CART: equal misclassification costs for false-positive and -negative results, and Gini splitting criterion in the analysis. $^{7}$

We used the decision tree from the CART analysis and information on the interrater reliability of the signs and symptoms of otitis media and our clinical judgement to create an algorithm that describes a practical approach to the diagnosis of otitis media.

We then validated this algorithm by applying it to a different dataset from a previously-conducted, large cohort study $(\mathrm{N}=783)$ that examined the efficacy of influenza vaccination in preventing AOM in a representative sample of children 6 to 24 months of age. ${ }^{8}$ This database will be referred to as the testing database. We selected one ear at random per child. We calculated the agreement between the diagnosis that would have been assigned if the algorithm had been used in the testing dataset and the diagnosis that was actually assigned by the otoscopists at the time of examination. Of the 7 otoscopists who participated in the training dataset, 3 also participated in the testing database.

\section{RESULTS}

Of the 264 children in the study, 263 children with complete data on signs and symptoms were included in the training dataset. Table 1 describes the symptoms and TM characteristics of these 263 children according to the 3 diagnostic categories. Findings that were significantly related to diagnosis $(P<.05)$ in the logistic regression model are indicated. All children with bulging TMs were diagnosed as having AOM; only $4(8 \%)$ children diagnosed as AOM had nonbulging TMs. Marked redness of the TM was found in $30 \%$ of children diagnosed as having AOM and $0 \%$ of children diagnosed as having OME or no effusion. Opacification was found in $100 \%$ of children diagnosed with AOM, 97\% of children diagnosed with OME, and $5 \%$ of children with no effusion. Normal mobility was found in $100 \%$ of children with no effusion, $32 \%$ of children with OME, and none of the children with AOM. Ear tugging was found in $44 \%, 24 \%$, and $7 \%$ of children with AOM, OME, and no effusion, respectively. Children with AOM were more likely than children with OME to exhibit irritability, ear pain, ear tugging, and to have bulging TMs, TM discoloration (yellow, white, amber, or blue), decreased mobility of the TM, and TM redness. When compared with children with no effusion, children 
Table 1. Signs and Symptoms According to Diagnosis in 263 Children Presenting For Primary Care

\begin{tabular}{|c|c|c|c|}
\hline & \multicolumn{3}{|c|}{$\begin{array}{c}\text { Training Dataset: } \\
\text { Pneumococcal Colonization } \\
\text { Study ( } \mathrm{N}=263) \text {, Number }(\%) \\
\text { of Children }\end{array}$} \\
\hline & $\begin{array}{c}\mathrm{AOM}^{*} \\
\mathrm{n}=50\end{array}$ & $\begin{array}{c}\text { OME } \\
\mathrm{n}=34\end{array}$ & $\begin{array}{c}\text { No Effusion } \\
\mathrm{n}=179\end{array}$ \\
\hline \multicolumn{4}{|l|}{ Symptoms present } \\
\hline Irritability† & $34(68)$ & $10(29)$ & $35(20)$ \\
\hline Feverł & $18(36)$ & $9(27)$ & $16(9)$ \\
\hline Ear paint & $17(34)$ & $2(6)$ & $4(2)$ \\
\hline Ear tugging十,‡ & $22(44)$ & $8(24)$ & $12(7)$ \\
\hline Playing less & $11(22)$ & $5(15)$ & $6(3)$ \\
\hline Eating lessł & $13(26)$ & $8(24)$ & $13(7)$ \\
\hline Disturbed sleep & $23(46)$ & $9(27)$ & $21(12)$ \\
\hline \multicolumn{4}{|l|}{ Signs present } \\
\hline Bulging TM† & $46(92)$ & 0 & 0 \\
\hline Opacified TM $\ddagger$ & $50(100)$ & $33(97)$ & $8(5)$ \\
\hline Discolored TM (not gray/pink)†, $\ddagger$ & $46(92)$ & $22(65)$ & 0 \\
\hline Decreased mobility of the TM†, $\neq$ & $50(100)$ & $23(68)$ & 0 \\
\hline Marked redness of the TM $†$ & $15(30)$ & 0 & 0 \\
\hline Air-fluid level†,‡ & $3(6)$ & $20(59)$ & 0 \\
\hline
\end{tabular}

${ }^{*} \mathrm{AOM}=$ acute otitis media; OME = otitis media with effusion; $\mathrm{TM}=$ tympanic membrane.

†AOM vs OME comparison on univariate analysis, $P<.05$. $\ddagger O M E$ vs no effusion comparison on univariate analysis, $P<.05$.

with OME were more likely to exhibit fever, ear tugging, decreased appetite, TM opacification, TM discoloration, decreased mobility of the TM, and an air-fluid level.

The decision tree describing the behavior of the otoscopists, which was derived by the use of recursive partitioning, is shown in Figure 1. Opacification and/or an air-fluid level, position, and marked redness of the tympanic membrane

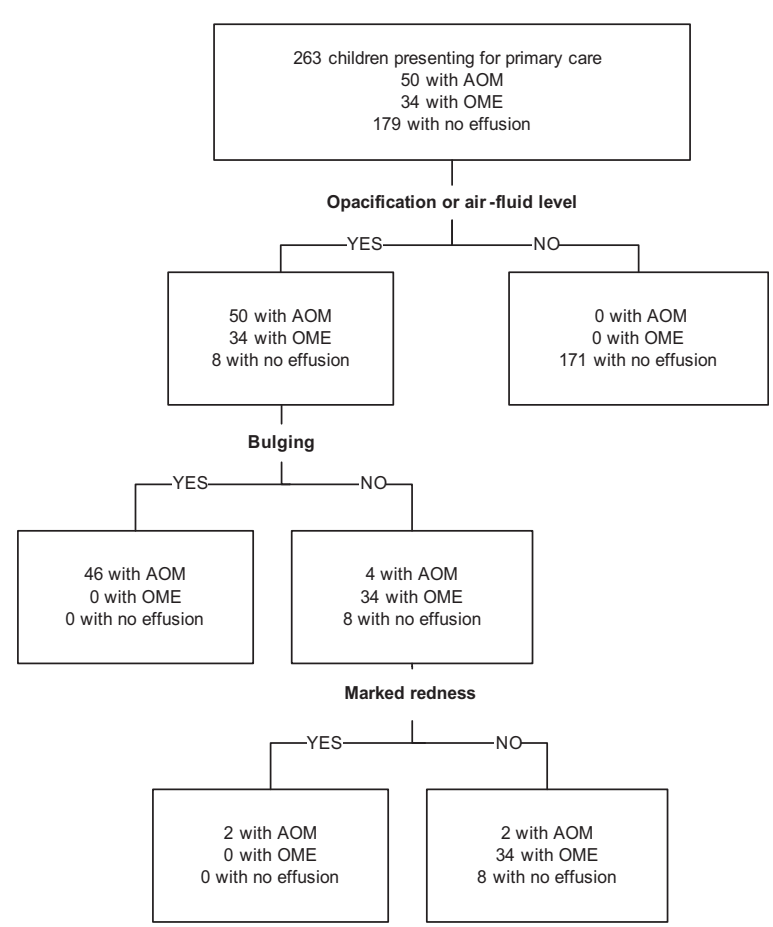

Figure 1. Decision tree for the diagnosis of otitis media (derived by the use of recursive partitioning).
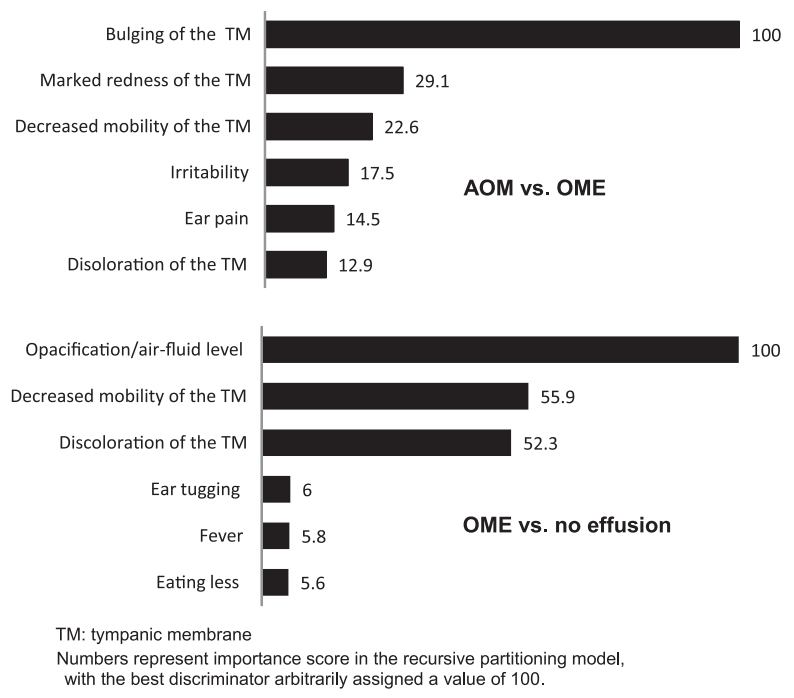

Figure 2. Relative importance of signs and symptoms in the diagnosis of otitis media.

were the findings that independently, and with the least amount of misclassification, best categorized children into the 3 diagnostic categories. However, isolated marked redness (without bulging) was used in only 2 cases $(<1 \%$ of all cases). None of the symptoms evaluated appeared in the decision tree.

The relative importance of the various findings in the diagnostic process is shown in Figure 2. Bulging of the $\mathrm{TM}$, followed distantly by marked redness of the TM, were the findings that otoscopists used to differentiate children with AOM from children with OME. Opacification of the TM and/or an air-fluid level, followed distantly by decreased mobility and discoloration of the TM, were the findings most useful in differentiating OME from no effusion.

The distribution of signs and symptoms in the testing dataset $(\mathrm{N}=783 \text {, one ear chosen at random per child })^{9}$ was similar to the distribution in the training dataset except that isolated marked redness of the TM (without bulging) was not observed. When the algorithm was applied to the testing dataset, it misclassified $8(1 \%)$ of cases; in 2 cases OME was misclassified as no effusion, in 3 cases no effusion was misclassified as OME, and in 3 cases AOM was misclassified as OME.

\section{Discussion}

Bulging of the TM was the main finding that the otoscopists in this study used to distinguish AOM from OME; the impact on diagnosis when findings other than bulging were considered was relatively minor. That the otoscopists here used the presence of bulging of the TM to diagnose $\mathrm{AOM}$, is not surprising; it is in accord with classic teaching. ${ }^{10-13}$ What is surprising is that bulging of the $\mathrm{TM}$ has not been required for eligibility in many recent clinical trials. ${ }^{3}$ The inclusion of large numbers of children without bulging TMs may have biased the results of these trials towards the null. In contrast, in recent placebocontrolled trials that have required bulging of the TM to diagnose AOM, investigators have reported much larger differences between treatment groups. ${ }^{14,15}$ Although we 


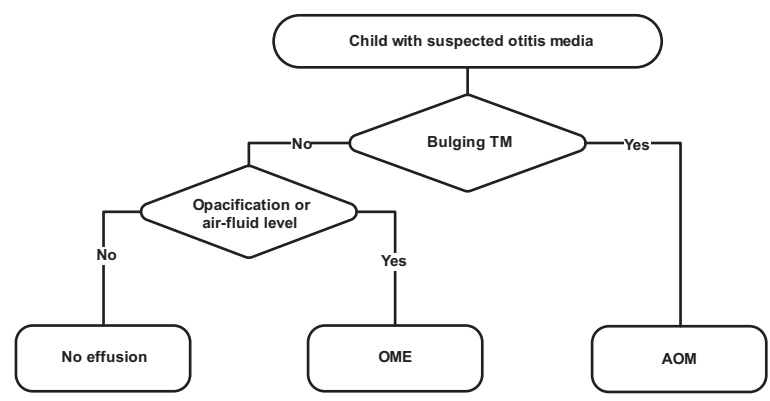

Figure 3. Proposed algorithm for the diagnosis of otitis media.

agree there is a spectrum of disease spanning the continuum between AOM and OME, most management strategies require that otitis media be classified clinically as either AOM or OME, a distinction that, because of the lack of a practical gold standard, is necessarily arbitrary. We suggest, in conformance with classic teaching, that the presence of bulging of the tympanic membrane be used to define AOM.

Marked redness of the TM was most often observed in conjunction with bulging of the TM. Although the otoscopists in this study appeared to use isolated marked redness of TM (without bulging) to establish the diagnosis of AOM in a small number of children (Fig. 2), we decided not to include it in our final algorithm (Fig. 3) for several reasons. First, trauma to the TM during removal of the cerumen may result in patches of marked erythema very similar to the inflammatory patches observed in children with AOM. Second, differentiating the distinct dark-red hemorrhagic patches observed in children with AOM from the diffusely pink or injected TM frequently observed in children without AOM can be challenging, especially for less-experienced otoscopists (Fig. 4). In fact, even among experienced otoscopists, the agreement regarding the presence or absence of marked redness is at best fair $(\kappa=0.32) .^{9}$ Third, isolated marked redness of the TM (without bulging) was present in less than $1 \%$ of children. Fourth, inclusion of marked redness in the algorithm would not have enhanced classification in the testing database (because isolated marked redness was not observed in the testing dataset). Because of these reasons, and because the intent of the algorithm is to present a general approach to the diagnosis of otitis media (rather than an exhaustive list all possible clinical scenarios where the diagnosis of
AOM could be justified), we decided not to include marked redness in the algorithm.

Otoscopists in this study primarily used opacification of the TM and/or the presence of an air-fluid level to differentiate children with OME from children with no effusion. Mobility and color of the TM, although helpful, were less influential in the establishing the diagnosis (Fig. 2). This finding suggests that in a large proportion of cases, ears with OME and ears with no effusion can be accurately distinguished by inspection alone; if structures of the middle ear are clearly visible and no airfluid levels are observed, OME is unlikely. This finding is consistent with our recent report in which we compared the diagnosis made by experienced otoscopists who examined children in the context of clinical care with the diagnosis made by a second independent group of otoscopists who viewed still TM images obtained by the first group of otoscopists. The agreement between the diagnoses (OME vs no effusion) assigned by the 2 groups of otoscopists was $85 \%$ despite one group being unaware of the mobility of the TM (or the presenting symptoms). ${ }^{9}$ Our data suggest that, in some children, mobility and color of the TM are useful in differentiating OME from no effusion. For example, some children have opacification of the TM without middle-ear effusion (eg, a child with scarred or thickened TMs). In these cases, mobility of the TM plays a central role in establishing the correct diagnosis. Accurate diagnosis of persistent $\mathrm{OME}$ is important because it determines the need for ventilating tube placement.

As compared with information provided through otoscopy (ie, the presence or absence of bulging), information regarding individual symptoms contributed relatively little to the diagnosis of AOM. This is in agreement with other recent studies. ${ }^{9,16}$ In a recent study, otoscopists with and without access to information regarding the child's presenting symptoms arrived at the same diagnosis $89 \%$ of the time. ${ }^{9}$ Because of the large overlap between symptoms of an upper respiratory infection and the symptoms of AOM, especially in preverbal children, it is not surprising that symptoms are not particularly useful in the diagnostic process. This is not to say that symptoms are unimportant. In the absence of symptoms, children would have not have been brought in for care. Furthermore, rapid resolution of symptoms is an important goal of

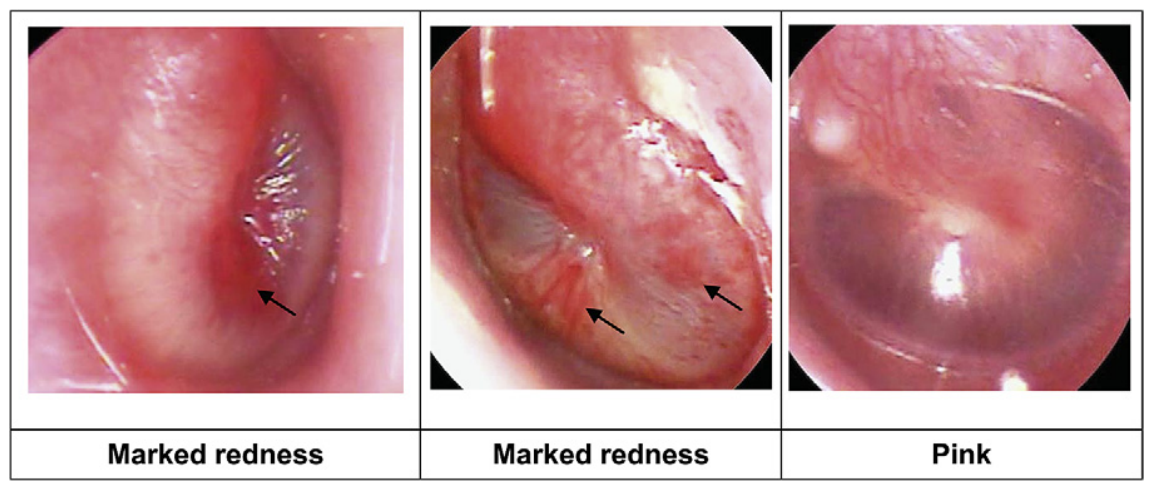

Figure 4. Marked redness of the TM (note distinct patches of intense erythema) compared with a diffusely pink TM. 
antimicrobial therapy. Careful monitoring of the symptoms of children in clinical trials remains an important method of assessing efficacy. ${ }^{4,6}$

The diagnostic approach suggested by our data differs from the approach endorsed by the 2004 AAP Clinical Practice Guideline in several important ways. ${ }^{1}$ We found that otalgia was not particularly helpful in differentiating AOM from OME (Fig. 2); rather, the clinicians involved more or less uniformly considered the presence of bulging as pathognomonic of AOM, and rarely diagnosed AOM in its absence.

The first limitation of this study relates to the overlap of some of the otoscopists in the testing and training datasets. The second and main limitation of this study is that it is purely descriptive; the algorithm developed provides a simple classification scheme that is consistent with the diagnostic process used by a particular group of otoscopists in Pittsburgh. Because of the lack of a practical reference standard, we cannot prove that those children diagnosed with AOM really had viable pathogens in their middle ears. In this respect, our study suffers from the same shortcomings as previously published research on the signs and symptoms of AOM.

Nevertheless, we believe that the data presented here are useful for several reasons. First, in previous studies researchers have attempted to understand the role of symptoms in the diagnosis of AOM by comparing it with the otoscopic diagnosis of AOM (ie, using otoscopy as the reference standard). ${ }^{17-19}$ By design, therefore, these studies were unable to assess the relative importance of the otoscopic signs versus the symptoms of AOM. Second, although a gold standard is available (tympanocentesis), it can ethically be performed only in children who are symptomatic and have otoscopic signs of otitis media. However, to evaluate the sensitivity and specificity of individual clinical findings, it would be necessary to perform tympanocentesis on patients with and without each of the signs and symptoms being evaluated. It was this lack of a practical gold standard that compelled us to use the approach used in this work. Third, although both the training and testing datasets were from studies conducted by our group in Pittsburgh, we previously demonstrated high levels of agreement between diagnoses assigned by our group and a second group of nationally recognized expert otoscopists. ${ }^{9}$

A caveat related to the interpretation of our data should be discussed to avoid future misinterpretation. The algorithm presented uses only 2 findings. This does not imply that all children can be correctly classified with the use of these 2 criteria. Our aim in creating an algorithm was not to identify the only findings that were important in the diagnosis. Indeed it would be difficult to examine the TM for opacification without at the same time noting discoloration or an air-fluid level. Rather, our objective was to identify a parsimonious model using the findings that could classify children with the least amount of misclassification, realizing that the additional information provided by other findings (eg, marked redness, mobility and color of the TM) could in some cases improve the diagnostic accuracy.

Our findings suggest that, under most circumstances, the diagnosis of AOM should be reserved for children who have a bulging TM, and they call into question clinical trials of the treatment of AOM in which TM bulging has not been a required element for participation.

\section{SUPPLEMENTARY DATA}

Supplementary data related to this article can be found online at doi:10.1016/j.acap.2012.01.007.

\section{REFERENCES}

1. American Academy of Pediatrics Subcommittee on Management of Acute Otitis Media. Diagnosis and management of acute otitis media. Pediatrics. 2004;113:1451-1465.

2. Hayden GF. Acute suppurative otitis media in children. Diversity of clinical diagnostic criteria. Clin Pediatr (Phila). 1981;20:99-104.

3. Shaikh N, Harvey K, Paradise JL, et al. The Cochrane Library and acute otitis media in children: an overview of reviews. EvidenceBased Child Health. 2009;4:390-399.

4. Shaikh N, Hoberman A, Paradise JL, et al. Development and preliminary evaluation of a parent-reported outcome instrument for clinical trials in acute otitis media. Pediatr Infect Dis J. 2009;28:5-8.

5. Kaleida PH, Stool SE. Assessment of otoscopists' accuracy regarding middle-ear effusion. Otoscopic validation. Am J Dis Child. 1992;146: $433-435$.

6. Shaikh N, Hoberman A, Paradise JL, et al. Responsiveness and construct validity of a symptom scale for acute otitis media. Pediatr Infect Dis J. 2009;28:9-12.

7. Breiman L, Friedman JH, Olshen RA, et al. Classification and Regression Trees. Monterey, Calif: Wadsworth, Inc.; 1984.

8. Hoberman A, Greenberg DP, Paradise JL, et al. Effectiveness of inactivated influenza vaccine in preventing acute otitis media in young children: a randomized controlled trial. JAMA. 2003;290:1608-1616.

9. Shaikh N, Hoberman A, Kaleida PH, et al. Otoscopic signs of otitis media. Pediatr Infect Dis J. 2011;30:822-826.

10. Hendley JO. Clinical practice. Otitis media. N Engl J Med. 2002;347: 1169-1174.

11. Holt L, McIntosh R. Holt's Diseases of Infancy and Childhood. 11th ed. New York: D. Appleton-Century Co; 1940:385.

12. Paradise JL. Otitis media in infants and children. Pediatrics. 1980;65: 917-943.

13. Paradise JL. On classifying otitis media as suppurative or nonsuppurative, with a suggested clinical schema. J Pediatr. 1987;111:948-951.

14. Hoberman A, Paradise JL, Rockette HE, et al. Treatment of acute otitis media in children under 2 years of age. $N$ Engl J Med. 2011; 364:105-115.

15. Tahtinen PA, Laine MK, Huovinen P, et al. A placebo-controlled trial of antimicrobial treatment for acute otitis media. N Engl J Med. 2011; 364:116-126.

16. Laine MK, Tahtinen PA, Ruuskanen O, et al. Symptoms or symptombased scores cannot predict acute otitis media at otitis-prone age. Pediatrics. 2010;125:e1154-e1161.

17. Heikkinen T, Ruuskanen O. Signs and symptoms predicting acute otitis media.[comment]. Arch Pediatr Adolesc Med. 1995;149:26-29.

18. Ingvarsson L. Acute otalgia in children-findings and diagnosis. Acta Paediatr Scand. 1982;71:705-710.

19. Niemela M, Uhari M, Jounio-Ervasti K, et al. Lack of specific symptomatology in children with acute otitis media. Pediatr Infect Dis. J. 1994;13:765-768. 